\title{
Effect of preoperative erector spinae muscles mass on postoperative outcomes in patients with left ventricular assist devices
}

\author{
Yukiharu Sugimura ${ }^{1}$, Nihat Sipahi ${ }^{2}$, Moritz Immohr ${ }^{3}$, Esma Yilmaz ${ }^{4}$, Joel Aissa ${ }^{5}$, Udo \\ Boeken $^{4}$, Hug Aubin ${ }^{6}$, Artur Lichtenberg ${ }^{5}$, Payam Akhyari ${ }^{7}$, and Hannan Dalyanoglu ${ }^{8}$ \\ ${ }^{1}$ Heinrich-Heine-Universitat Dusseldorf \\ ${ }^{2}$ Universitatsklinikum Dusseldorf \\ ${ }^{3}$ Heinrich Heine University Düsseldorf \\ ${ }^{4}$ Dep. of Cardiovascular Surgery \\ ${ }^{5}$ University Hospital Dusseldorf \\ ${ }^{6}$ Heinrich-Heine-University Düsseldorf \\ ${ }^{7}$ Medical Faculty, Heinrich-Heine University \\ ${ }^{8}$ Affiliation not available
}

May 24, 2021

\begin{abstract}
Background: Frailty influences the postoperative outcomes in patients undergoing left ventricular assist device (LVAD) implantation; however, a quantitative evaluation method has not been established. The purpose of the present study was to evaluate whether preoperative ESM mass is associated with short- and long-term clinical outcomes in patients with LVAD. Methods: A total of 119 consecutive patients with LVAD were enrolled between January 2010 and October 2017 at a single heart center. The ESM index and Hounsfield units (HU) of the ESM were calculated by computed tomography for preoperative ESM mass evaluation. We then statistically evaluated the in-hospital mortality, major adverse cardiovascular events (MACE), duration of hospital stay, and long-term survival. Results: In a multivariate Cox regression analysis, ESM index and HU of the ESM indicated no effect on the in-hospital mortality, MACE, and long-term survival. In addition, the ESM index presented a weak but significant negative linear correlation only with the duration of hospital stay $(r=-0.21, p<0.05)$. In contrast, the model for end-stage liver disease (MELD) score and preoperative venous-arterial extracorporeal membrane oxygenation (va-ECMO) were significant predictive factors for in-hospital mortality (MELD score: $\mathrm{p}<0.001$, hazard ratio [HR] 1.1; preoperative va-ECMO: $\mathrm{p}<0.01$, HR 2.72) and MACE (MELD score: $\mathrm{p}<0.001$, HR 1.07; preoperative va-ECMO: $\mathrm{p}<0.005$, HR 2.62). Conclusion: Preoperative ESM mass might predict the length of hospital stay in patients undergoing LVAD implantation. In contrast, it had no effect on MACE, in-hospital mortality, or long-term survival in this study.
\end{abstract}

\section{Introduction}

Frailty is a geriatric concept that describes frail older adults and is regarded as a medical syndrome with a poor prognosis ${ }^{1}$. The main manifestation of frailty is a decrease in skeletal muscle mass, i.e., sarcopenia associated with aging. However, in chronic heart failure, it is often caused by secondary sarcopenia due to complex pathological conditions, such as cardiac cachexia. Frailty has been associated with the prognosis of patients with heart failure (HF), suggesting that it may be a new prognostic indicator that reflects the clinical outcomes in these patients.

With advancements in medical technology, more HF patients have undergone left ventricular assist device (LVAD) implantation, and their clinical outcomes have also improved. With the expected expansion of 
LVAD indications, it is necessary to determine the parameters indicative for LVAD implantation in frailty. In other words, it is indispensable to preoperatively distinguish whether the frailty of HF patients will be recoverable after LVAD implantation by improving their hemodynamics. However, it has not been well established whether patients with severe frailty will respond to LVAD implantation. Thus, the development of criteria to assess preoperative frailty in HF patients is expected to provide an appropriate indication for LVAD implantation and encourage efforts to prevent unnecessary LVAD implantation, which is not negligible in medical economics.

This study examines the functionality of the erector spinae muscle (ESM) mass, which can be assessed using chest computed tomography $(\mathrm{CT})$. This is usually performed in the context of the preoperative evaluation for LVAD implantation. ESM mass would be an advantageous prognostic marker, allowing the preoperative assessment of the precise individual patient benefit before the LVAD operation. Here, we analyzed the effect of the preoperative mass of the ESM on the clinical outcomes of LVAD patients with HF in our cohort.

\section{Methods}

\section{Ethics committee approval}

This retrospective data analysis was approved by the Ethics Committee of the Medical Faculty of the Heinrich-Heine-University Düsseldorf (Ref. 2020 - 832).

\section{Study design and data collection}

This retrospective study analyzed 168 consecutive patients with LVAD between January 2010 and October 2017 at a single heart center. Patients who did not undergo preoperative chest CT evaluations were excluded from this study. In total, 119 patients were enrolled in the study. The purpose of this study was to examine the ability of preoperative ESM mass as a predictive factor for postoperative clinical outcomes in patients undergoing LVAD implantation. To realize this purpose, we provided two endpoints in this study. The primary endpoint was the clinical outcome at hospital discharge, and the secondary endpoint was the postoperative follow-up status up to December 2019 (766 \pm 889 days, median 372 days), in which patients who had already undergone heart transplantation (HTX) were also considered to be "living after LVAD implantation," similar to other LVAD patients. Within the category of the primary endpoint, we analyzed the in-hospital mortality, major adverse cardiovascular events (MACE), and the duration of hospital stay. On the other hand, at the secondary endpoint, only the survival rate in the follow-up periods was analyzed. The collected patient data were pseudonymized and arranged in numerical order. As a preoperative liver function assessment, the model for end-stage liver disease (MELD) score was evaluated based on the following formula; $3.78 \times$ (bilirubin) + $11.2 \times$ (international normalized ratio $)+9.57 \times($ creatinine $)+6.43$. In this study, MACE was defined as a postoperative cerebral vascular event, a cardiac event including operative or interventional procedures, or cardiac death.

\section{Surgical indication and procedure}

Indications for LVAD implantation were determined based on the European Association for Cardio-Thoracic Surgery guidelines. Three therapy concepts were documented in this study: "bridge to transplant," "bridge to candidacy," and "destination therapy." All patients underwent LVAD implantation via sternotomy. In 31 patients $(26.1 \%)$, the LVAD was implanted without a heart-lung machine (HLM), i.e., the off-pump technique, whereas in 88 patients $(73.9 \%)$, it was implanted with HLM. For intracorporeal LVAD, we implanted three different devices: HeartWare HVAD (Medtronic, Minneapolis, MN), Heartmate (HM) 2 (Abbott, Inc., Chicago, IL, USA), and HM 3. Only one patient underwent paracorporeal LVAD implantation using EXCOR (Berlin Heart, Inc., The Woodlands, TX, USA). Concomitant operations were performed in 11 patients: aortic valve replacement (AVR), $\mathrm{n}=3$; atrial septum defect closure, $\mathrm{n}=3$; left atrial appendage closure, $\mathrm{n}=2 ; \mathrm{AVR}+\mathrm{LAA}$ closure, $\mathrm{n}=1 ; \mathrm{AVR}+$ tricuspid valve repair, $\mathrm{n}=1$; and another $\mathrm{n}=1$. Despite these differences associated with surgical indications and procedures, all 119 patients were considered to be homogeneous cases for the analysis in this study.

\section{CT-based analysis of ESM mass}


CT-based radiological measurements were an essential part of this study. As in a previous study, the crosssectional areas of bilateral ESM were performed on a single axial chest CT image at the level of the lower margin of the 12th thoracic vertebrae ${ }^{2-5}$. In this study, we used the ESM index as per the formula $=$ ESM area/body mass index (BMI), which was analyzed for the quantification of ESM areas, according to the patient's physics. Furthermore, Hounsfield units (HU) of the ESM were determined to assess the density of the ESM.

\section{Statistical analysis}

All statistical analyses were administrated with the Statistical Package for Social Sciences $\mathbb{R} 25.0$ software for Mac OS X® (IBM, Chicago, USA). Utilizing this program, descriptive and comparative statistics (i.e., $\chi^{2-}$ test, t-test, Mann-Whitney U-test) were performed. The significance tests were performed bilaterally. The data of the interval-scaled variables are expressed as mean \pm standard deviation. Multivariate data analysis was performed using Cox proportional hazards regression to determine the perioperative risk factors for clinical outcomes in the two endpoints of this study. Receiver-operating characteristic (ROC) analysis was performed to evaluate the accuracy of the statistical performance for some variables. Statistical correlation analysis was performed using Spearman's correlation coefficient. Statistical significance was set at $\mathrm{P}<0.05$.

\section{Results}

\section{Patient characteristics}

The patient characteristics of all cohorts are shown in Table 1 . Ninety-nine (83.2\%) men and 20 (18.6\%) women were enrolled at the time of surgery, with a mean age of $55.9 \pm 11.9$ years at the time of surgery. The mean BMI of all patients was within the normal range $\left(26.5 \pm 4.98 \mathrm{~kg} / \mathrm{m}^{2}\right)$. In our cohort, 59 patients $(49.6 \%)$ were categorized in the interagency registry for mechanically assisted circulatory support (INTERMACS) 1. Similarly, 52 patients $(43.7 \%)$ required preoperative venous-arterial extracorporeal membrane oxygenation (va-ECMO) support. This means that half of our cohort suffered from critical cardiogenic shock and needed definitive permanent mechanical circulatory support. Because of these severely impaired patient cohorts, the mean MELD score was also relatively high $(17.2 \pm 8.33)$. This effect was also reflected in the postoperative clinical outcomes, i.e., $43.7 \%$ of postoperative va-ECMO support or $40 \%$ of right ventricular assist device support. Forty-four patients (37\%) had MACE, whereas the in-hospital mortality rate was $27.7 \%$. Regarding the long-term outcome, Figure 1 shows the Kaplan-Meier survival curve for up to years 10 post-implant, in which the survival rate rapidly decreased up to 1 year post-implant (52.6\%). Subsequently, this trend became gradual. The survival rate at 10 years post-implant was $31.5 \%$.

\section{Cox proportional hazards regression for preoperative parameters}

For multivariable Cox regression analysis, age, BMI, INTERMACS profile 1, history of cardiopulmonary resuscitation, baseline disease, preoperative va-ECMO, creatinine, MELD score, ESM index, and HU of the ESM were evaluated (Table 2) . Regarding the in-hospital mortality and MACE, the MELD score, as well as the preoperative va-ECMO implantation, were significant predictive factors in this study (in-hospital mortality: MELD score, $\mathrm{p}<0.001$, hazard ratio [HR] 1.1, confidence interval [CI] 1.05-1.14; preoperative vaECMO, $\mathrm{p}<0.01$, HR 2.72, CI 1.32-5.59. MACE: MELD score, $\mathrm{p}<0.001$, HR 1.07, CI 1.03-1.11; preoperative va-ECMO, p < 0.005, HR 2.62, CI 1.39-4.90). However, no predictive factors were found for the follow-up survival rate. ESM mass, i.e., ESM index and HU of the ESM, indicated no effect on the postoperative clinical outcomes.

\section{Effect of preoperative ESM mass on hospital stay}

Excluding 33 in-hospital mortalities, we analyzed the correlation between preoperative ESM mass and the duration of hospital stay in 86 patients. Overall, the ESM index presented a weak but significant negative linear correlation with the duration of hospital stay, whereas the HU of the ESM did not indicate a significant correlation (ESM index; $\mathrm{r}=-0.21, \mathrm{p}<0.05, \mathrm{HU}$ of the ESM, $\mathrm{p}<0.33$ ) (Figure 2 ).

\section{ROC analysis of ESM mass for clinical outcomes}


To evaluate the accuracy of the statistical performance, we performed a ROC analysis of ESM mass for clinical outcomes. The area under the curve of ESM mass, i.e., the ESM index and HU of the ESM, was less than 0.5 for evaluating all clinical outcomes in this study (Figure 3a-c ).

\section{Discussion}

The main purpose of the present study was to identify whether short- and long-term postoperative outcomes after LVAD implantation were related to preoperative ESM mass. Pre-LVAD-implantation frailty might affect postimplant mortality ${ }^{6}$. Regarding the evaluation method of frailty, the condition of the skeletal muscles might reflect the patients' frailty, i.e., sarcopenia. Their predictive potential for clinical outcomes was examined in this study. In the setting of LVAD implantation, it was demonstrated that the quantity of the pectoralis major muscle provides predictive information on the clinical outcomes after LVAD implantation 7,8 . However, many authors have reported that antigravity muscles might reflect the patients' frailty more effectively in predicting postoperative outcomes ${ }^{5,9}$. Thus, we focused on the ESM as a predictor of postoperative outcomes in patients with LVAD in our evaluation.

In contrast to our hypothesis, a smaller ESM mass was not confirmed as the main determinant of in-hospital mortality, MACE, and follow-up mortality in this study. In recent years, the ESM has often been evaluated in the field of pneumology as a useful prognostic factor for assessing clinical outcomes. Decreased physical activity due to lung dysfunction, e.g., in chronic obstructive pulmonary disease, pulmonary fibrosis, infection, and even post-lobectomy status causes muscle weakness in the antigravity muscles, which predicts the clinical prognosis of such patients ${ }^{2,4,5,10}$. Interestingly, a study of patients who underwent liver transplantation due to end-stage liver disease also reported that the paraspinal muscle index, i.e., the combined mass index of the psoas muscle and ESM, was an independent predictive factor for early posttransplant survival in male patients $^{3}$. Regarding the effectiveness of antigravity muscle analysis as a predictive preoperative factor for the postoperative outcomes in patients with LVAD, Heberton et al. demonstrated that sarcopenia patients defined by the psoas muscle area showed a numeric tendency of higher mortality and longer hospital stay without significant difference in each clinical outcome, whereas sarcopenia was an independent predictive factor in patients who suffered from either higher mortality or had longer hospital stays ${ }^{11}$. The authors concluded that a low psoas muscle area might represent a higher mortality or longer hospital stay risk in patients with LVAD. This is similar to our result that a smaller ESM index proved to be an independent risk factor for a longer hospital stay in this study.

Moreover, we demonstrated that the MELD score and preoperative va-ECMO implantation are risk factors for MACE and in-hospital mortality, respectively. Regarding the MELD score, this issue has already been reported by our team ${ }^{12}$. We suppose that this is the key reason why skeletal muscle evaluation, for example, of the e.g., ESM or the psoas muscle, would not allow speculation on the clinical outcomes in LVAD patients. This means that patients undergoing LVAD implantation who already have visceral organ decompensation have a poor prognosis regardless of the manifestation of muscle atrophy.

Thus, we conclude that ESM mass is not associated with acute severe clinical outcomes, such as MACE or in-hospital mortality. In contrast, the ESM may be used to estimate the length of hospital stay of patients who survive the acute phase after LVAD operations. Furthermore, ESM mass did not predict the long-term survival outcome because of the multifactorial complication risks in LVAD patients.

This study had several limitations. First, this was a retrospective analysis with a limited cohort size of nonrandomized patients. Second, this was a single-center study, meaning that potential systemic measurement errors and factors could influence the outcomes. Third, the patients undergoing LVAD implantation were heterogeneous in their characteristics, whereas we analyzed all patients as a homogenous cohort in this study. The subanalysis depending on more defined categories, e.g., baseline disease or disease status, and even the type of LVAD devices, provides a more detailed insight into the confirmation of our conclusions. Therefore, further studies are required.

\section{Conclusion}


The evaluation of preoperative ESM mass might predict the length of hospital stay in patients undergoing LVAD implantation. However, preoperative ESM has no effect on short-term severe adverse clinical outcomes, such as MACE or in-hospital mortality and long-term survival. To the best of our knowledge, this is the first report to analyze the preoperative ESM mass in patients undergoing LVAD.

\section{Author contributions}

Conception of the work: Yukiharu Sugimura, Hannan Dalyanoglu

Analysis and interpretation of data: Yukiharu Sugimura, Hannan Dalyanoglu

Drafting of the manuscript: Yukiharu Sugimura

Critical revision of the manuscript: Nihat Firat Sipahi, Moritz Benjamin Immohr, Esma Yilmaz, Udo Boeken, Hug Aubin, Artur Lichtenberg, Payam Akhyari, Hannan Dalyanoglu

Responsibility for treatment decisions: Artur Lichtenberg

Supervision: Joel Aissa, Artur Lichtenberg, Payam Akhyari

\section{Acknowledgements}

We gratefully acknowledge the work of the members of our department, who engaged with this study.

\section{References:}

1. Morley JE, Vellas B, van Kan GA, et al. Frailty consensus: a call to action. J Am Med Dir Assoc. 2013;14(6):392-397.

2. Tanaka A, Komiya K, Yamasue M, et al. Quantitative assessment of the association between erector spinae muscle and in-hospital mortality in elderly patients with pulmonary tuberculosis. BMC Res Notes.2021;14(1):134.

3. Pinto Dos Santos D, Kloeckner R, Koch S, et al. Sarcopenia as prognostic factor for survival after orthotopic liver transplantation.Eur J Gastroenterol Hepatol. 2020;32(5):626-634.

4. Nakano A, Ohkubo H, Taniguchi H, et al. Early decrease in erector spinae muscle area and future risk of mortality in idiopathic pulmonary fibrosis. Sci Rep. 2020;10(1):2312.

5. Tanimura K, Sato S, Fuseya Y, et al. Quantitative Assessment of Erector Spinae Muscles in Patients with Chronic Obstructive Pulmonary Disease. Novel Chest Computed Tomography-derived Index for Prognosis.Ann Am Thorac Soc. 2016;13(3):334-341.

6. Cooper LB, Hammill BG, Allen LA, et al. Assessing Frailty in Patients Undergoing Destination Therapy Left Ventricular Assist Device: Observations from Interagency Registry for Mechanically Assisted Circulatory Support. ASAIO J. 2018;64(1):16-23.

7. Cogswell R, Trachtenberg B, Murray T, et al. A Novel Model Incorporating Pectoralis Muscle Measures to Predict Mortality After Ventricular Assist Device Implantation. J Card Fail.2020;26(4):308-315.

8. Teigen LM, John R, Kuchnia AJ, et al. Preoperative Pectoralis Muscle Quantity and Attenuation by Computed Tomography Are Novel and Powerful Predictors of Mortality After Left Ventricular Assist Device Implantation. Circ Heart Fail. 2017;10(9).

9. Ikezoe T, Mori N, Nakamura M, Ichihashi N. Effects of age and inactivity due to prolonged bed rest on atrophy of trunk muscles.Eur J Appl Physiol. 2012;112(1):43-48.

10. Miller JA, Harris K, Roche C, et al. Sarcopenia is a predictor of outcomes after lobectomy. $J$ Thorac Dis. 2018;10(1):432-440. 
11. Heberton GA, Nassif M, Bierhals A, et al. Usefulness of Psoas Muscle Area Determined by Computed Tomography to Predict Mortality or Prolonged Length of Hospital Stay in Patients Undergoing Left Ventricular Assist Device Implantation. Am J Cardiol. 2016;118(9):1363-1367.

12. Maxhera B, Albert A, Ansari E, Godehardt E, Lichtenberg A, Saeed D. Survival predictors in ventricular assist device patients with prior extracorporeal life support: selecting appropriate candidates.Artif Organs. 2014;38(9):727-732.

\section{TABLE 1. Clinical characteristics in 119 patients undergoing LVAD implantation}

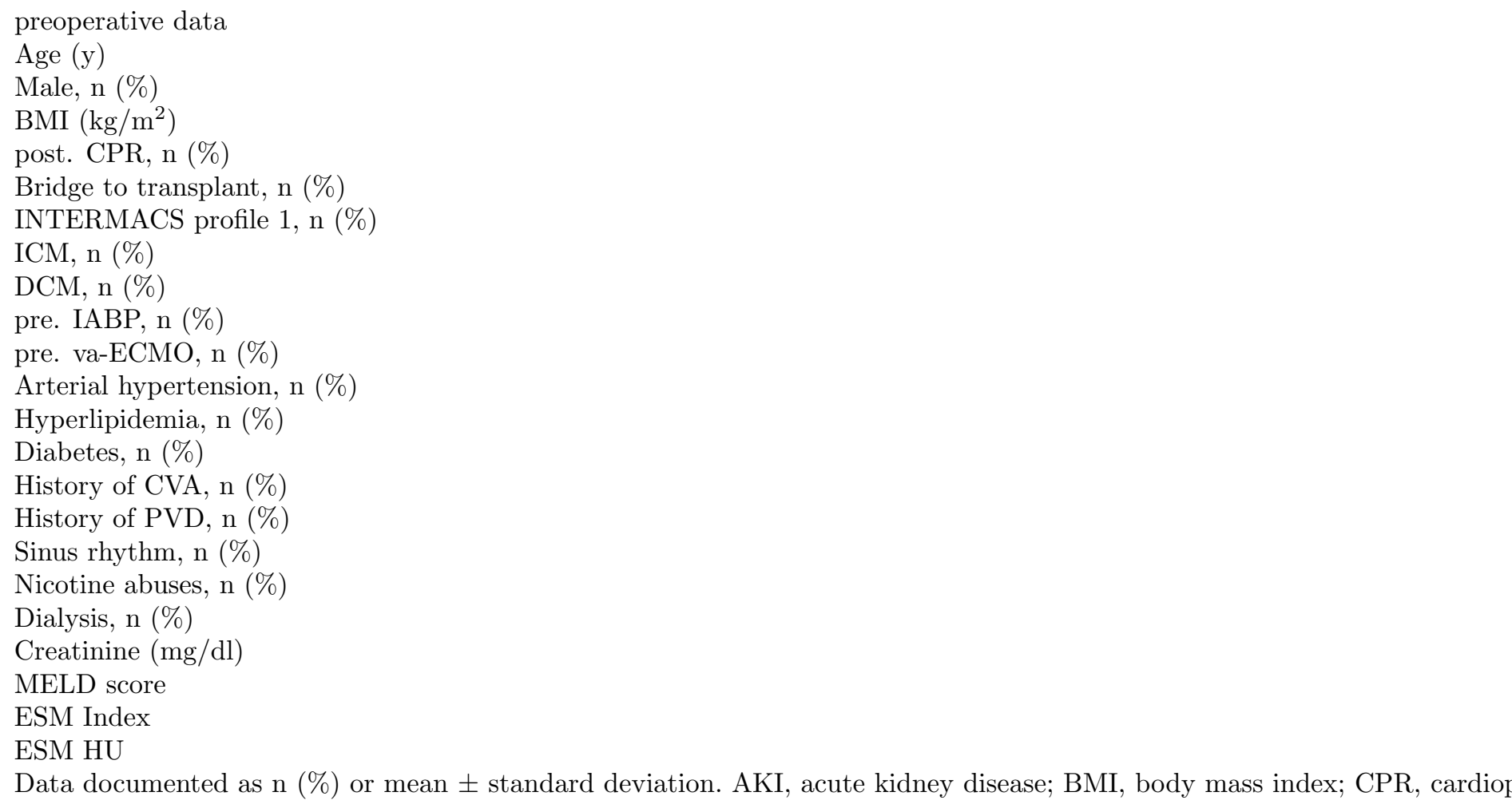

TABLE 2. Multivariate Cox hazard analysis of postoperative clinical outcomes

in-hospital mortality

MELD score

pre. va-ECMO

MACE

MELD score

pre. va-ECMO

CI, confidence interval; HR, hazard ratio; MACE, major adverse cardiac event; MELD, model of end-stage liver disease; pre

\section{Figure legends}

Figure 1. Long-term survival in 119 patients undergoing left ventricular assist device implantation

Figure 2. The correlation graphic between preoperative ESMI and the duration of hospital stay in 86 
patients excluding 33 in-hospital mortality. ESMI, erector spinae muscle index.

Figure 3. receiver-operating characteristic analysis of ESM mass for each clinical outcome. (a) in-hospital mortality, (b) the major adverse cardiovascular events, and (C) long-term survival. ESM, erector spinae muscle; HU, Hounsfield units.

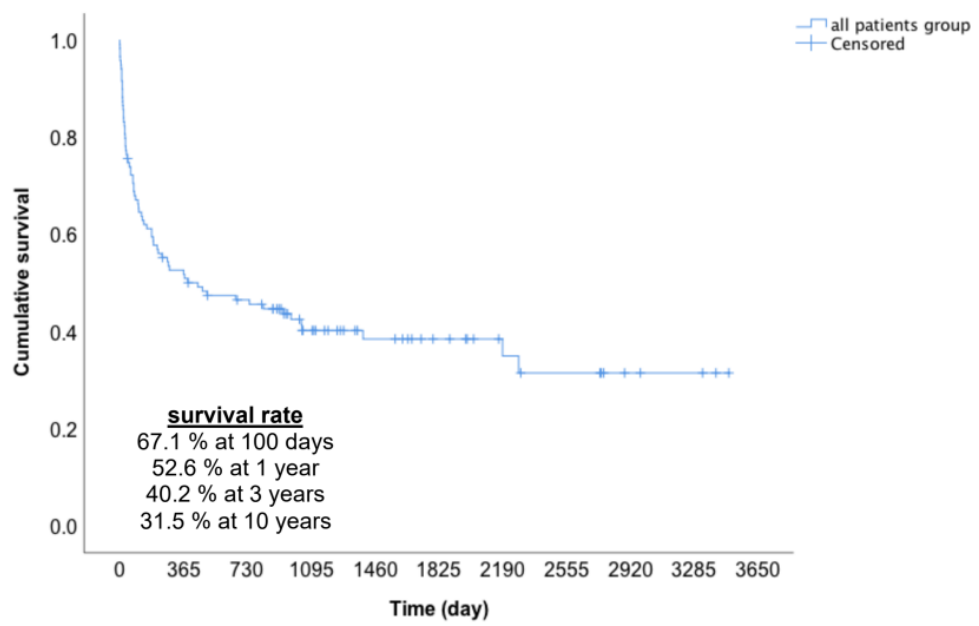




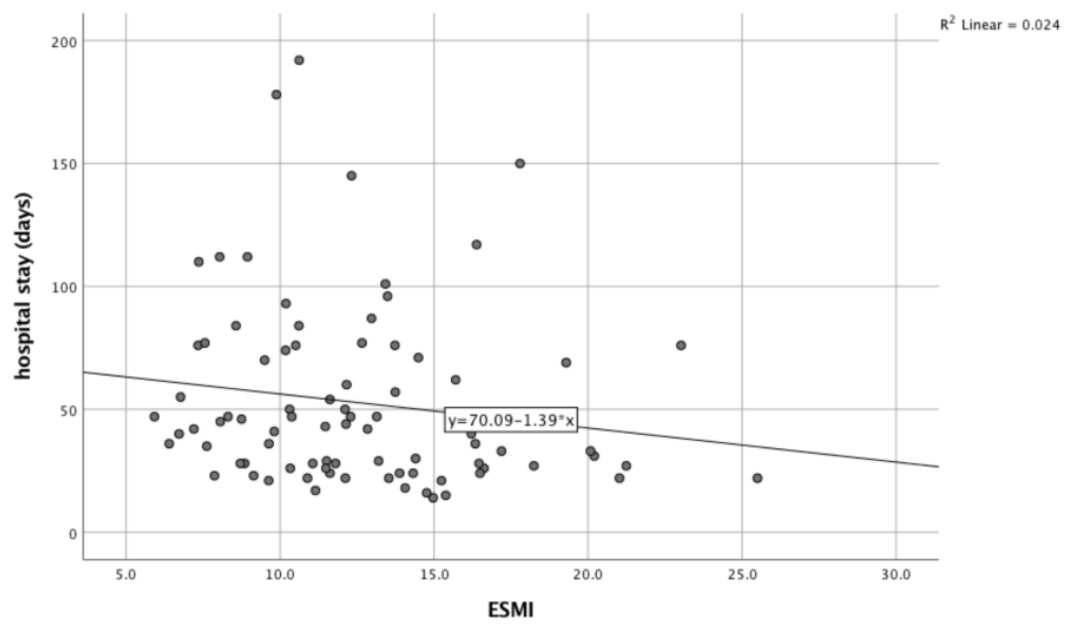




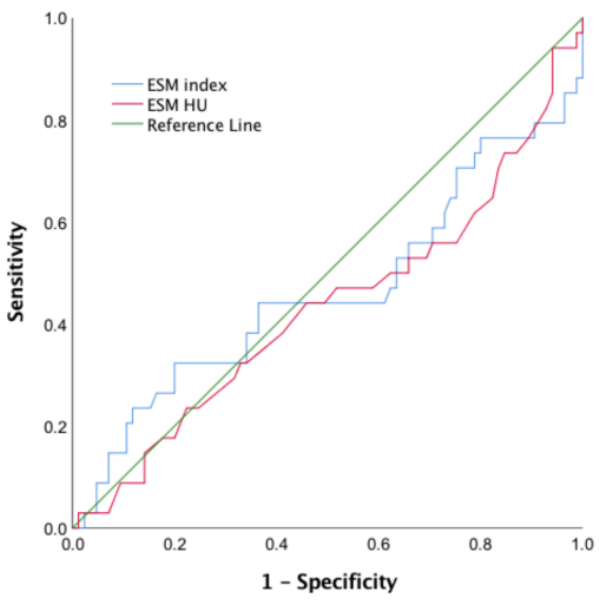




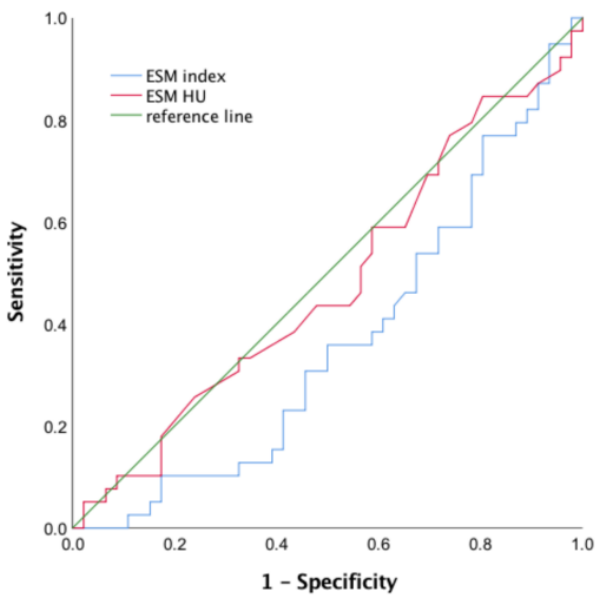




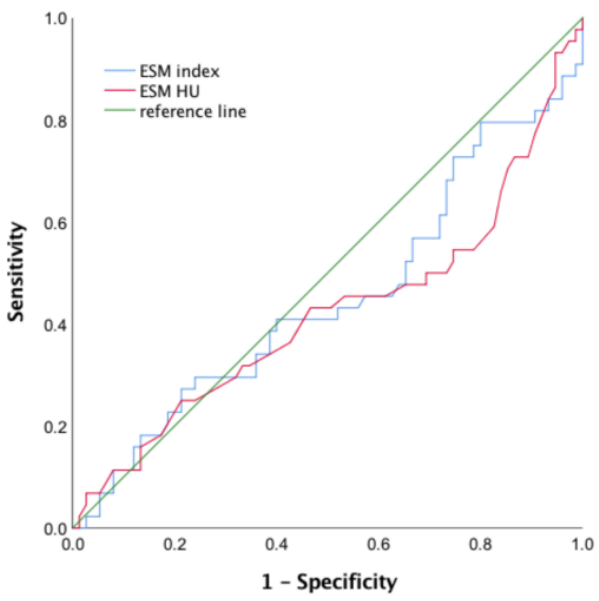

